\title{
Sofrimento psíquico vivenciado por mulheres diante do diagnóstico de câncer de mama: uma revisão bibliográfica reflexiva
}

\author{
Psychological suffering experienced by women facing the diagnosis of breast cancer: a \\ reflective literature review
}

Sufrimiento psíquico experimentado por mujeres que enfrentan el diagnóstico de cáncer de seno: una revisión reflexiva de la literatura

Ana Paula Lopes ${ }^{1 *}$, Carmen Aparecida Cardoso Maia Camargo ${ }^{1}$, Maria Ambrosina Cardoso Maia1.

\section{RESUMO}

Objetivo: Verificar como o sofrimento psíquico é vivenciado por mulheres com diagnóstico de câncer de mama. Revisão bibliográfica: Nota-se, de maneira geral, uma diminuição na qualidade de vida global dessas mulheres, com constante sentimento de medo, de insegurança e de ansiedade. Sendo o câncer de mama uma doença com consequências desfigurantes, há uma alteração da percepção corporal e de sua identidade como mulher, representados pela perda do seio e do cabelo, ambos símbolos de feminilidade. Ademais, os relacionamentos dessas pacientes sofrem alterações: há o receio do abandono do conjugal, a perda da fertilidade e do poder da escolha de uma nova gestação, e o aumento da necessidade de uma rede de apoio social. Considerações finais: Conclui-se que as mulheres com câncer de mama possuem, para além da doença física que enfrentam, diversos desafios psicológicos: as alterações no corpo e em suas relações e seus medos as tornam especialmente suscetíveis à depressão e ao sofrimento psicológico. No tratamento delas, não pode se desconsiderar a existência desses, de suas inseguranças e de sua saúde mental, devendo ser implementado um tratamento que aborde todos esses aspectos.

Palavras-Chave: Câncer de mama, Sofrimento psíquico, Mastectomia.

\section{ABSTRACT}

Objective: To verify how psychological suffering is experienced by women diagnosed with breast cancer. Bibliographic review: In general, there is a decrease in the overall quality of life of these women, with a constant feeling of fear, insecurity, and anxiety. As breast cancer is a disease with consequences that modify their bodies, there is a change in the body's perception and their identity as a woman, represented by breast and hair loss, both signals of femininity. In addition, the relationships of these patients suffer changes: there is the fear of abandonment by couples, loss of fertility and the possibility of choosing a new pregnancy and increased need for a social support network. Final considerations: It's possible to conclude that women with breast cancer, in addition to the physical disease, face several psychological challenges such as: changes in their body and in their relationships and their fears, are especially susceptible to depression and psychological suffering. In their treatment, they can't disregard the existence of their safety and mental health, and a treatment should be implemented encompassing all these aspects.

Key words: Breast cancer, Psychological distress, Mastectomy.

\section{RESUMEN}

Objetivo: Verificar cómo las mujeres diagnosticadas con cáncer de seno experimentan el sufrimiento psicológico. Revisión bibliográfica: En general, hay una disminución en la calidad de vida general de estas mujeres, con un sentimiento constante de miedo, inseguridad y ansiedad. Como el cáncer de seno es una enfermedad con consecuencias desfigurantes, hay un cambio en la percepción e identidad del cuerpo como

1Universidade do Estado de Minas Gerais (UEMG), Passos - MG. *E-mail: lopessanap@gmail.com 
mujer, representada por la pérdida del seno y el cabello, ambos símbolos de la feminidad. Además, las relaciones de estos pacientes sufren cambios: existe el temor de abandonar la relación matrimonial, la pérdida de fertilidad y el poder de elegir un nuevo embarazo, y una mayor necesidad de una red de apoyo social. Consideraciones finales: Se concluye que las mujeres con cáncer de seno tienen, además de la enfermedad física que enfrentan, varios desafíos psicológicos: los cambios en el cuerpo, sus relaciones y sus miedos las hacen especialmente susceptibles a la depresión y el sufrimiento psicológico. En su tratamiento, la existencia de estos, sus inseguridades y su salud mental no se pueden ignorar, y se debe implementar un tratamiento que aborde todos estos aspectos.

Palabras clave: Cáncer de mama, Distrés psicológico, Mastectomía.

\section{INTRODUÇÃO}

O câncer representa o conjunto de doenças que têm em comum o crescimento desordenado de células, com a consequente invasão de tecidos e órgãos, determinando a formação de tumores malignos, que podem se espalhar para outras regiões do corpo - ou seja, que podem gerar metástases. A heterogeneidade dos cânceres pode ser observada pelas variadas manifestações clínicas e morfológicas, diferentes assinaturas genéticas e diversas respostas às terapêuticas administradas (INCA, 2019a).

O câncer de mama é o segundo tipo de tumor mais frequente do mundo e o mais frequente entre as mulheres, excluindo-se o câncer de pele. Representou $29 \%$ dos novos casos de câncer no Brasil a cada ano - no mundo, o percentual é de $25 \%$-, sendo esperados cerca de 60.000 novos casos no país (INCA, 2019b).

Existem diversas opções para o tratamento dessa doença, indicados com base no quadro clínico, histórico e resposta da paciente: radioterapia, quimioterapia, cirúrgico (mastectomia total ou quadrantectomia), hormonioterapia, entre outros. O tratamento pode ser tanto curativo quanto paliativo, a depender do estágio da doença e do retorno diante da terapêutica adotada (SOCIEDADE BRASILEIRA DE MASTOLOGIA, 2017).

Quando diagnosticado e tratado precocemente, o câncer de mama apresenta um bom prognóstico, na grande maioria dos casos (SOCIEDADE BRASILEIRA DE MASTOLOGIA, 2017), mas, ainda assim, o diagnóstico pode suscitar pensamentos negativos, fomentados pela possibilidade de um prognóstico ruim e a incerteza do desenrolar da doença (MATTIAS SR, et al., 2018).

O tratamento quimio e radioterápico pode ser fonte de dor, estresse e diminuição da autoestima; enquanto o tratamento cirúrgico pode provocar diversas repercussões psicológicas, com impacto negativo na autoimagem corporal, na funcionalidade da mulher, nos seus relacionamentos conjugais - tanto afetivo quanto sexual - e em outras diversas áreas de sua vida (PEREIRA GB, et al., 2017; RODRIGUES NS, et al., 2017).

Estudos apontam que a primeira preocupação da mulher após receber o diagnóstico é a sua sobrevivência. Em seguida, outras preocupações surgem, entre elas destacam-se: a possibilidade de existência de metástases, a mutilação e desconfiguração do seu corpo, as possíveis consequências para a sua vida sexual, mudanças nos hábitos e atividades diárias e possíveis limitações que irão a acompanhar após o tratamento (FURTADO HMS, et al., 2016).

Ademais, a incerteza suscitada pela avalanche de informações enfrentada diante do diagnóstico, somada à necessidade de enfrentar a sensação de finitude, são também motivos de preocupação para essas pacientes (DOLINA JV, et al., 2014).

As mulheres são lembradas a todo momento de sua fragilidade e da possível - e, em suas concepções, provável - morte próxima, tanto pelo ambiente hospitalar (uma constante durante todo o período de tratamento), pelos amigos e familiares (que sempre contam histórias sobre os piores desfechos possíveis para os quadros dessas mulheres), quanto por elas próprias, que já imaginam qual é o quadro de maior calamidade em suas próprias doenças. 
Cada paciente possui uma experiência única do adoecimento pelo câncer de mama, porém nota-se que o suporte e apoio familiar, assim como o convívio com um grupo social, auxiliam as pacientes a lidarem com esse sofrimento psicológico a que as pacientes estão submetidas, ajudando-as a se adaptarem à nova realidade (ARAUJO MR, et al., 2016).

Frente a esses dados, e visto a perpetuação da relevância do tema evidenciada pelos altos índices de casos de câncer de mama em mulheres, acredita-se na importância de abordar essa temática, influenciando e embasando a multidisciplinariedade e pluralidade do tratamento dessas pacientes, melhorando sua condição de vida.

Esse estudo tem por objetivo, portanto, verificar como o sofrimento psíquico é vivenciado por mulheres com diagnóstico de câncer de mama.

\section{REVISÃO BIBLIOGRÁFICA}

\section{Categoria 1 - Sofrimento psíquico relacionado ao medo do câncer e à qualidade de vida}

O medo é uma emoção comumente relatada pelas pacientes com câncer de mama. O temor está presente desde o momento da suspeita do diagnóstico, havendo uma tendência da diminuição deste após a radioterapia. Os efeitos colaterais crônicos e progressivos, contudo, são muitas vezes interpretados como um lembrete constante do câncer, podendo até mesmo ser mal interpretados como um sinal da recorrência da malignidade (YANG Y, et al., 2018).

Importante frisar que, apesar da equipe multiprofissional saber reconhecer os efeitos colaterais crônicos apenas como isso, e não qualquer sinal de recorrência da doença, a paciente está com receio, insegura e qualquer sintoma é motivo de grande angústia e dúvida. É papel da equipe acolher as vulnerabilidades dessa mulher e acalmá-la de forma a tentar a todo momento melhorar a qualidade de vida dessa paciente por meio dessas ações. Qualquer queixa que se demonstre significativa para a mulher, deve ser também significativa para a equipe (ainda que clinicamente não tenha tanta significância), especialmente considerando-se que a paciente é um ser biopsicossocial e deve ser abordada por todas essas vertentes.

Um dos aspectos recorrentes é a existência de uma fase de negação, tanto de sintomas quanto do diagnóstico, que reduz os efeitos deletérios da relação da pessoa com seu corpo e sua mente. Geralmente é transitória e necessária para a proteção da cognição da mulher (SILVA RM, et al., 2018). A fase da negação também é encontrada em diversos outros processos enfrentados durante a vida, como o luto, e é importante o profissional considerá-la como parte crucial do amadurecimento da condição, não forçando ou querendo privar a mulher desse momento.

Diversos problemas encontrados pelas pacientes - medo da morte, destruição de seus planos de vida, mudança na aparência física, dificuldades financeiras, ansiedade - contribuem para uma diminuição da qualidade de vida de maneira global (SALIBASIC M e DELIBEGOVIC S, 2018). Deve-se salientar que as adversidades não são encontradas de forma isoladas, complicando a abordagem dos mesmos.

Como exemplo, pode-se citar que as dificuldades financeiras acentuadas pelo diagnóstico do câncer de mama - gastos decorrentes do tratamento, como custeio do transporte e alimentação, ou perda de benefícios no trabalho, entre outros são os principais motivos dessa dificuldade (FIREMAN KM, et al., 2018) - podem estar associadas à apreensão de perder o parceiro, especialmente considerando-se que os problemas financeiros por si só, sem a doença, já se mostram como um frequente motivo de atribulação nos relacionamentos atuais.

Alguns estudos demonstram que mulheres submetidas a cirurgia conservadora tendem a ter níveis maiores de satisfação com a vida quando comparadas às que fizeram mastectomia (CIPORA E, et al., 2018) e que pacientes com a autoimagem menos alterada apresentam resultados melhores na reabilitação e melhor prognóstico de qualidade de vida (FIREMAN KM, et al., 2018); porém não é consenso, visto que outros estudos não encontraram efeitos significativos do tipo de tratamento cirúrgico na qualidade de vida (SALIBASIC M e DELIBEGOVIC S, 2018). 
Esses fatores - o medo e a diminuição da qualidade de vida - adicionados às mudanças da aparência física, da rotina e dos relacionamentos tornam as mulheres com diagnóstico de câncer de mama mais suscetíveis à depressão, especialmente as que enfrentam cirurgia radical (SALIBASIC M e DELIBEGOVIC $\mathrm{S}, 2018)$.

Outros importantes fatores preditores de qualidade de vida nessa situação são idade, nível de educação, status de emprego/desemprego e estágio do câncer (CHEN Q, et al, 2018). Depois do tratamento, de modo geral, o bem-estar das mulheres tende a melhorar (VILLAR RR, et al., 2017), porém algumas sequelas e efeitos colaterais crônicos do tratamento, como cansaço, fadiga, dores, limitação dos movimentos do membro superior do mesmo lado que a mastectomia foi performada e prejuízos na habilidade motora, podem repercutir permanentemente nas atividades diárias e profissionais da mulher, influenciando diretamente na sua funcionalidade e qualidade de vida pós-tratamento (PENNA GB, et al., 2017).

É importante considerar, portanto, a condição do câncer de mama como um fator de risco tanto para o transtorno depressivo maior quanto para outros distúrbios depressivos, realizando uma abordagem ampliada que seja capaz de acolher essas mulheres, realizar prevenção e, também, perceber os primeiros sinais e sintomas, para um diagnóstico e tratamento precoces. A saúde mental delas deve ser tratada com tanta prioridade quanto a saúde física nesse momento, visto que as consequências futuras podem ser extensivas em ambas as situações.

\section{Categoria 2 - Sofrimento psíquico relacionado à sua percepção corporal}

Imagem corporal é a estrutura que inclui a percepção do corpo como um todo e suas partes individuais, seu movimento e limitações. Para as mulheres, também inclui seu sentimento de feminilidade e de ser e se sentir atraente (MIAJA M, et al., 2017).

A representação da mama na sociedade moderna, estando associada à construção da feminilidade da mulher e sendo também símbolo de sua sexualidade, fertilidade e maternidade, torna essa uma parte do corpo da mulher essencial na construção da sua identidade (SÁ GS e PINHEIRO-CAROZZO NP, 2018). A valorização da mama feminina mostra-se presente em diversos âmbitos sociais: na moda, com o uso de decotes ou exploração de diversos modelos que a valorizem, por exemplo, até mesmo para a amamentação, com o surgimento de diversas campanhas sobre a amamentação em locais públicos.

Não é equívoco afirmar, portanto, que, sendo o câncer de mama uma doença que se relaciona com as identidades das mulheres, ele é capaz de alterar e prejudicar a percepção das pacientes com sua própria imagem (CHEN Q, et al., 2018). A doença é muitas vezes enfrentada como um "defeito no corpo", por isso é sentida como um grande impacto em sua beleza e feminilidade (KARIMI SE, et al., 2018).

Estudos demonstram uma angústia persistente e severa das pacientes relacionada a imagem corporal, principalmente nas mulheres que não podem realizar a reconstrução mamária (HUBBELING HG, et al., 2018). O tratamento cirúrgico do câncer de mama está significativamente conectado ao modo como as pacientes percebem seu corpo, porém tanto a mastectomia quanto o tratamento conservador têm risco similar de baixa autoestima (JABŁOŃSKI MJ, et al., 2018).

Para além das alterações relacionadas com a perda do seio, também foram relatadas como significativas a perda de cabelo, presença de cicatrizes cirúrgicas, mudanças de peso e linfedema (MIAJA M, et al., 2017). A queda do cabelo representa a perda de outro símbolo da feminilidade, que é ainda mais visível que a própria mama, e é relatada como uma dificuldade da doença, embora muitas pacientes tenham receio em transparecer esse sentimento, especialmente por ser considerado 'supérfluo' ou 'insignificante', diante da possibilidade de morte se não realizar o tratamento (OLIVEIRA TR, et al., 2019).

A perda dos cabelos como consequência do tratamento oncológico é amplamente divulgada e conhecida, já sendo esperado pela maioria das pacientes. Entretanto, o conhecimento da perda dos pelos das demais partes do corpo - sobrancelha, pelos axilares, das pernas e púbicos - não é tão público, e é uma surpresa para a maioria das mulheres com câncer de mama. Para melhor enfrentamento da alopecia, elas tendem a se apegar na condição da perda do cabelo como um 'mal necessário', utilizando da premissa 
de atribuição de sentido à um acontecimento dentro da noção e construção de um 'bem maior' (REIS APA e GRADIM CVC, 2018).

Embora a insatisfação com sua imagem corporal possa ser observada também em mulheres não diagnosticadas com câncer de mama, demonstra-se que as que possuem esse diagnóstico são mais insatisfeitas que as demais (PRATES ACL, et al., 2017). A presença da baixa autoestima, associada aos escores de imagem corporal mais baixos, está relacionada a sintomas depressivos (BOING L, et al., 2019).

Considerando a grande incidência de insatisfação com a imagem corporal nas mulheres com câncer de mama, é importante que a abordagem multiprofissional trabalhe esse aspecto durante o tratamento e consultas com essas pacientes. A reconstrução deve ser discutida e analisada, assim que possível, assim como o apoio das decisões das mulheres.

A perda do cabelo, sendo uma grande questão não só para as pacientes com câncer de mama, mas para as pacientes oncológicas de modo geral, deve ser tratada com naturalidade, porém deve-se respeitar a decisão da mulher, após compartilhar as opções viáveis e discuti-las com as mulheres. Auxiliar no processo para adoção do visual careca, ou incentivar o uso de acessórios ou perucas, dependendo da escolha da paciente, é também papel da equipe multiprofissional no atendimento à essas mulheres.

\section{Categoria 3 - Sofrimento psíquico relacionado a alterações nos relacionamentos}

Com o diagnóstico do câncer de mama, essas mulheres diagnosticadas enfrentam alterações profundas em seus relacionamentos, tanto conjugais, quanto maternais e/ou sociais. As pacientes relatam deterioração na experiência de relações íntimas com o parceiro, tanto pela perda do atributo de sua feminilidade (estando incluso tanto os seios quando cabelo) quanto pela presença de disfunções sexuais em decorrência do tratamento (JABŁOŃSKI MJ, et al., 2018).

Entre os principais domínios de disfunções sexuais relatadas, encontram-se: interesse sexual, excitação, orgasmo, frequência e habilidade de relaxar, entre outras. Um fator de maior preocupação é a possível persistência desses sintomas no período após o tratamento, o que acontece com a maioria das pacientes (MIAJA M, et al., 2017).

Não banalizar a preocupação em relação a vida sexual das pacientes é importante, pois pode ser um fator considerável para a diminuição da qualidade global de vida, já discutida anteriormente, na categoria 1 deste trabalho. É papel da equipe multiprofissional acolher esse - e todas os demais - receios da paciente, mesmo que seja na atual etapa do tratamento, apenas tomar ciência, acalmar a paciente e garantir que essa queixa não será esquecida; retomando-a em momento mais propicio.

Junto ao receio de perder o apelo sexual, encontra-se relatos de medo de perder o companheiro (KARIMI SE et al, 2018). Embora na maioria dos casos os relatos sejam de resiliência e crescimento do casal, há aqueles com angústia, ansiedade, má comunicação e abandono emocional e físico, que fragilizam e provocam maior sofrimento nas mulheres (HUBBELING HG, et al., 2018).

Assim como discutido sobre o pensamento frequente em relação ao pior desfecho da doença possível, aqui também pode acontecer isso. A mulher, em sua insegurança e fragilidade diante de sua nova condição, pode ser negativamente impactada e, mesmo que o companheiro não dê indícios ou não tenha qualquer atitude que demonstre intenção de terminar o relacionamento, esse medo pode estar presente e ser uma grande fonte de insatisfação para a paciente.

Durante esse momento de instabilidade, representado pela mudança de toda sua rotina e planejamento de vida, as histórias de abandono ouvidas durante todos seus anos de vida podem tomar uma grande proporção e ganhar força, contribuindo para a perpetuação do medo, angústia e ansiedade.

Estar sem um parceiro durante o enfrentamento da doença está relacionado com menores índices de qualidade de vida (GANGANE N, et al, 2018), principalmente considerando-se que as maiores fontes de apoio relatadas pelas pacientes são, na maioria dos casos, o companheiro e os filhos (ZHANG $\mathrm{H}$, et al., 2018). 
Acerca dos filhos e de sua família, para aquelas mulheres que já possuem sua própria, há uma tendência das pacientes de se preocuparem com eles acima de si próprias, tentando ao máximo postergar a transição de seu antigo papel "provedor" para o seu novo "papel doente" (HUBBELING HG, et al., 2018). Importante frisar que na cultura moderna a mulher ainda é valorizada pelo seu papel de mãe e pela sua responsabilidade de cuidadora e de organizadora do lar, sendo então um aspecto de sofrimento para ela abrir mão desse papel, mesmo que parcialmente e provisoriamente (SILVA RM, et al., 2018).

Outro dos problemas enfrentado pelas mulheres é a destruição de seus planos de vida, já que muitas relatam o desejo que tinham de ter filhos no momento do diagnóstico, o que é impossibilitado pela frequente perda de fertilidade consequente ao tratamento (SALIBASIC M e DELIBEGOVIC S, 2018). A infertilidade pode estar muitas vezes relacionada a sintomas depressivos e angústia persistente (HUBBELING HG, et al., 2018).

A perda do poder de escolha é um fator importante. Mesmo que filhos não sejam um projeto de vida a curto prazo, o simples fato de se tornar não mais uma escolha, mas uma imposição a impossibilidade da gestação, é um motivo de angústia. A sondagem do desejo de engravidar no momento do diagnóstico pela equipe multiprofissional $e$, mais do que isso, a identificação das pacientes que se mostrem mais angustiadas pelo efeito adverso da infertilidade decorrente do tratamento, é essencial e deve ser realizado logo no momento do diagnóstico e a investigação deve também ocorrer durante as etapas posteriores do tratamento.

Uma rede de apoio social é um fator essencial para a melhora da qualidade de vida das mulheres com câncer de mama, porém muitas vezes é percebido um isolamento social, principalmente pelo medo da divulgação de seu estado como doente (HUBBELING HG, et al., 2018). A rede de apoio envolve tanto família quanto amigos, e, mais importante que o simples apoio, é um que não a trate como a doente ou a incapaz, que a amizade e o tratamento desprendido antes do adoecimento não sejam de todo modificados após o diagnóstico.

Por esse motivo, muitas vezes as amizades mais significativas para as pacientes durante esse período são aquelas que se iniciam com outras mulheres com o mesmo diagnóstico e que enfrentam as mesmas dificuldades, que passam a se conhecer, em muitas ocasiões, na própria sala de espera durante o período de tratamento. Atividades e dinâmicas em sala de espera poderiam ser uma estratégia interessante para ajudar a promover e incentivar esse contato entre essas mulheres, em semelhança com aquelas que são desenvolvidas atualmente na atenção primária à saúde, respeitando suas particularidades.

\section{CONSIDERAÇÕES FINAIS}

Ressalta-se a presença de sofrimentos psíquicos em diversos aspectos da vida da mulher com câncer de mama, passando pelo constante medo que o diagnóstico traz, a destruição de seus planos de vida, insatisfação com a imagem corporal, baixa autoestima, deterioração da experiência de relações sexuais, até o receio em perder o apelo sexual. As mulheres com câncer de mama possuem, portanto, para além da doença física que enfrentam, diversos desafios psicológicos: as alterações no corpo, de suas relações e seus medos as tornam especialmente suscetíveis à depressão e ao sofrimento psicológico. Na intervenção direcionada à elas, não pode ser desconsiderada a existência desses, de suas inseguranças e de sua saúde mental, devendo ser implementado um tratamento multiprofissional que aborde todos esses aspectos, não negligenciando as nuances psicológicas suscitadas pela condição; assim como devem ser incentivados maiores estudos na área para o desenvolvimento de abordagens cada vez mais completas, precisas e benéficas às pacientes, pilar central de todo $o$ atendimento.

\section{AGRADECIMENTOS E FINANCIAMENTO}

Este artigo é parte integrante do projeto de pesquisa "Estudo sobre o sofrimento psíquico vivenciado pelas mulheres diante do diagnóstico de câncer de mama", financiado pelo Programa Institucional de Apoio à Pesquisa (PAPq) da Universidade do Estado de Minas Gerais (UEMG), aprovado pelo comitê de ética sob o parecer número 3.535.413, e desenvolvido pela acadêmica Ana Paula Lopes, sob orientação de Carmen Aparecida Cardoso Maia Camargo e coorientação de Maria Ambrosina Cardoso Maia e Luciana Helena Silva Sarturino. 


\section{REFERÊNCIAS}

1. ARAUJO MR, et al. As consequências da mastectomia: enfoque físico e psicológico. Fisioterapia Ser, 2016; 11(4):188-194.

2. BOING L, et al. Factor associated with depression symptoms in women after breast cancer. Revista de Saúde Pública, 2019; 53:30.

3. CHEN Q, et al. Health-related quality of life among women breast cancer patients in Eastern China. Biomed Research International, 2018; 2018:1452635.

4. CIPORA E, et al. Surgical method of treatment and level of satisfaction with life among women diagnosed with breast cancer, according to time elapsed since performance of surgery. Annals of Agricultural and Environmental Medicine, $2018 ; 25(3): 453-459$.

5. DOLINA JV, et al. Diferentes temporalidades no adoecimento por câncer de mama. Revista da Escola de Enfermagem da USP, 2014; 48(Esp2):77-84.

6. FIREMAN KM, et al. Percepção das Mulheres sobre sua Funcionalidade e Qualidade de Vida após Mastectomia. Revista Brasileira de Cancerologia, 2018; 64(4):499-508.

7. FURTADO HMS, et al. Repercussões do diagnóstico de câncer de mama feminino para diferentes faixas etárias. Ciência \& Saúde, 2016; 9(1):8-14.

8. GANGANE N, et al. Quality of life determinants in breast cancer patients in Central Rural India. Asian Pacific Journal of Cancer Prevention, 2018; 18(12):3325-3332.

9. HUBBELING HG, et al. Psychosocial needs of young breast cancer survivors in Mexico City, Mexico. PLoS One, 2018; 13(5):e0196931.

10. Câncer de mama. 2019a. In: Instituto Nacional de Câncer (INCA). Disponível em: https://www.inca.gov.br/tipos-decancer/cancer-de-mama. Acesso em: 09 jul. 2019.

11. O que é câncer? 2019b. In: Instituto Nacional de Câncer (INCA). Disponível em: https://www.inca.gov.br/o-que-ecancer. Acesso em: 09 jul. 2019.

12. JABŁOŃSKI MJ, et al. The relationship between surgical treatment (mastectomy vs. breast cancer conserving treatment) and body acceptance, manifesting femininity and experiencing an intimate relation with a partner in breast cancer patients. Psychiatria Polska, 2018; 52(5):859-872.

13. KARIMI SE, et al. Identifying the social determinants of breast health behavior: a qualitative contente analysis. Asian Pacific Journal Cancer Prevention, 2018; 19(7):1867-1877.

14. MATTIAS SR, et al. Câncer de mama: sentimentos e percepções das mulheres diante do diagnóstico. Revista Online de Pesquisa, 2018; 10(2):385-390.

15. MIAJA M, et al. Psychological impact of alterations in sexuality, fertility, and body image in young breast cancer patients and their partners. Revista de Investigación Clínica, 2017; 69(4):204-209.

16. OLIVEIRA TR, et al. Câncer de mama e imagem corporal: impacto dos tratamentos no olhar de mulheres mastectomizadas. Saúde e Pesquisa, 2019; 12(3):451-462.

17. PENNA GB, et al. Repercussões do tratamento cirúrgico do câncer de mama sobre a propriocepção, sensibilidade e funcionalidade. Fisioterapia Brasil, 2017; 18(2):197-204.

18. PEREIRA GB, et al. Impacto do tratamento do câncer de mama na autoimagem e nos relacionamentos afetivos de mulher mastectomizadas. LifeStyle Journal, 2019; 4(1):99-119.

19. PRATES ACL, et al. Influence of body image in women undergoing treatment for breast cancer. Revista Brasileira de Ginecologia e Obstetrícia, 2017; 39(4):175-183.

20. REIS APA, GRADIM CVC. A Alopecia no Câncer de Mama. Revista de Enfermagem UFPE Online, 2018; 12(2):447455.

21. RODRIGUES NS, et al. Importância do acompanhamento psicológico em mulher mastectomizada: artigo de revisão. Arquivos Catarinenses de Medicina, 2017; 46(1):164-172.

22. SÁ GS, PINHEIRO-CAROZZO NP. Imagem Corporal e Habilidades Sociais em pacientes com câncer de mama. Revista de Psicologia da IMED, 2018; 10(1):37-55.

23. SALIBASIC M, DELIBEGOVIC S. The quality of life and degree of depression of patients suffering from breast cancer. Medical Archives, 2018; 72(3):202-205.

24. SILVA RM, et al. Mastectomy and the meaning attributed by patients to delay in early diagnosis of breast cancer. Asian Pacific Journal Cancer Prevention, 2018; 19(8):2083-2088.

25. SOCIEDADE BRASILEIRA DE MASTOLOGIA. Regional Piauí. Câncer de mama: consenso da Sociedade Brasileira de Mastologia - Regional Piauí. Teresina: EDUFPI; 2017; 328p.

26. VILLAR RR, et al. Quality of life and anxiety in women with breast cancer before and after treatment. Revista LatinoAmericana de Enfermagem, 2017; 25:e2958.

27. YANG $Y$, et al. Fear of cancer recurrence trajectory during radiation treatment and follow-up into survivorship of patients with breast cancer. BMC Cancer, 2018; 18:1002.

28. ZHANG H, et al. Experiences of social support among chinese women with breast cancer: a qualitative analysis using a framework approach. Medical Science Monitor, 2018; 24:574-581. 